\title{
Power Optimization of a Miller Thermal Cycle with respect to Residual Gases and Equivalence Ratio
}

\author{
R. EBRAHIMI* \\ Department of Agriculture Machine Mechanics, Shahrekord University \\ P.O. Box 115, Shahrekord, Iran \\ (Received September 6, 2012; in final form March 15, 2013)
}

\begin{abstract}
The performance of an air standard Miller cycle is analyzed using finite-time thermodynamics. The relations between the power output and the compression ratio and between the power output and the thermal efficiency are derived by detailed numerical examples. The results show that, throughout the compression ratio range, the power output decreases with increasing residual gases. The results also show that if compression ratio is less than certain value, the power output decreases with increasing equivalence ratio, while if compression ratio exceeds certain value, the power output first increases and then starts to decrease with increasing equivalence ratio. The conclusions of this investigation are of importance when considering the designs of actual Miller engines.
\end{abstract}

DOI: $10.12693 /$ APhysPolA.124.6

PACS: 05.70.Ln, 82.60.Fa, 88.05.-b, 88.20.td

\section{Introduction}

Since finite-time thermodynamics $[1-4]$ is a powerful tool for the performance analysis and optimization of real internal combustion engine cycle, much work has been performed for the performance analysis and optimization of finite time processes and finite size devices $[5,6]$. In 1940s, Miller [7] proposed a different Otto cycle with unequal compression and expansion stroke called the Miller cycle. the Miller cycle has been put attention recently [8], and some authors have examined the finite-time thermodynamic performance of the Miller cycle. Hatamura et al. [9] report that the Miller cycle has advantages such as higher mean effective pressure than the Otto cycle with lower nominal compression ratio. Fukuzawa et al. [10] described the main technologies and performance specifications for a high efficiency Miller cycle gas engine as well as for the series of engines planned in the future.

Al-Sarkhi et al. [11] compared the performance characteristic curves of the Atkinson cycle with those of the Miller and Joule-Brayton cycles by using numerical examples, and outlined the effect of maximizing power density on the performance of the cycle efficiency.

Wu et al. [12] performed a performance analysis and optimization of a supercharged Miller cycle Otto engine. Their zero-dimensional analysis is based on Otto cycle. They report that the Miller cycle shows no efficiency advantage and suffers a penalty in power output in the normally aspirated version. In the supercharged Otto engine adopted for the Miller cycle, it has again no efficiency advantage but does provide increased output with a reduced propensity to engine knock problem.

Ge et al. derived the performance characteristics of the Miller cycle with heat transfer loss [13] and with heat

*e-mail: Rahim.Ebrahimi@gmail.com transfer and friction-like term losses [14], respectively. These works were done without considering the variable specific heats of working fluid. Ge et al. [15] also studied the effect of variable specific heats of working fluid on the performance of endoreversible the Miller cycle.

Al-Sarkhi et al. [16] presented theoretical investigations into the Miller cycle engine performance, studying the influence of the main engine design variables and system irreversibilities. Chen et al. [17] built a class of generalized irreversible universal steady flow heat engine cycle model consisting of two heating branches, two cooling branches, and two adiabatic branches with consideration of the losses of heat resistance, heat leakage, and internal irreversibility.

The performance characteristics of Diesel, Otto, Brayton, Atkinson, dual and Miller cycles were derived. Ebrahimi studied the performance of reversible Miller cycle. The normalized network output and normalized thermal efficiency are obtained by introducing the compression ratio, the expansion-compression ratio and the specific heat ratio. Ebrahimi [18] also investigated the effects of the relative air-fuel ratio and the stroke length on the performance of an irreversible Atkinson cycle. As can be seen in the relevant literature, the investigations of the effects of residual gases and equivalence ratio on the performance of the Miller cycle do not appear to have been published. Therefore, the objective of this study is to examine the effects of residual gases and equivalence ratio on the performance of air standard Miller cycle.

\section{Thermodynamic analysis}

The pressure-volume $(P-V)$ and the temperatureentropy $(T-S)$ diagrams of an irreversible Miller heat engine is shown in Fig. 1 , where $T_{1}, T_{2}, T_{3}, T_{4}$, and $T_{5}$ are the temperatures of the working substance in state points $1,2,3,4$, and 5 . Process $1 \rightarrow 2$ is a reversible isentropic compression. The heat addition occurs in the constant volume process $2 \rightarrow 3$. Process $3 \rightarrow 4$ is a reversible 
adiabatic expansion. The heat rejection occurs in two steps: processes $4 \rightarrow 5$ and $5 \rightarrow 1$ are constant volume and constant pressure heat rejections, respectively.

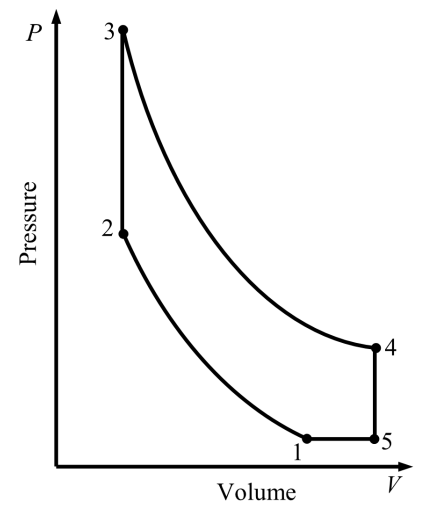

Fig. 1. $P-V$ diagram for the air standard Miller cycle.

The relations between the mass flow rate of the fuel $\left(\dot{m}_{\mathrm{f}}\right)$ and the mass flow rate of the air $\left(\dot{m}_{\mathrm{a}}\right)$, between $\dot{m}_{\mathrm{f}}$ and the mass flow rate of the air-fuel mixture $\left(\dot{m}_{\mathrm{t}}\right)$ are defined as [18, 19]:

$$
\dot{m}_{\mathrm{a}}=\phi^{-1} \dot{m}_{\mathrm{f}}\left(m_{\mathrm{a}} / m_{\mathrm{f}}\right)_{\mathrm{s}}
$$

and

$$
\dot{m}_{\mathrm{t}}=\frac{\dot{m}_{\mathrm{f}}\left[\phi+\left(\frac{m_{\mathrm{a}}}{m_{\mathrm{f}}}\right)_{\mathrm{s}}\right]}{\phi\left(1-x_{\mathrm{r}}\right)},
$$

where $m_{\mathrm{a}} / m_{\mathrm{f}}$ is the air-fuel ratio and the subscript $\mathrm{s}$ denotes stoichiometric conditions, $\phi$ is the equivalence ratio and $x_{\mathrm{r}}$ is the residual fraction from the previous cycle, and can find it as 190]:

$$
x_{\mathrm{r}}=m_{\mathrm{r}} /\left(m_{\mathrm{a}}+m_{\mathrm{f}}+m_{\mathrm{r}}\right),
$$

where $m_{\mathrm{r}}$ is the mass of residual gases. It should be noted here that the residual gases were assumed to consist of $\mathrm{CO}_{2}, \mathrm{H}_{2} \mathrm{O}$, and $\mathrm{N}_{2}$.

The mass specific heat at constant volume for the working fluid $\left(C_{\mathrm{vt}}\right)$ is defined as:

$$
\begin{aligned}
& C_{\mathrm{vt}}= \\
& \frac{\left(1-x_{\mathrm{r}}\right)\left[\left(\frac{m_{\mathrm{a}}}{m_{\mathrm{f}}}\right)_{\mathrm{s}} C_{\mathrm{va}}+\phi C_{\mathrm{vf}}\right]+x_{\mathrm{r}} C_{\mathrm{vr}}\left[\phi+\left(\frac{m_{\mathrm{a}}}{m_{\mathrm{f}}}\right)_{\mathrm{s}}\right]}{\phi+\left(\frac{m_{\mathrm{a}}}{m_{\mathrm{f}}}\right)_{\mathrm{s}}}
\end{aligned}
$$

where $C_{\mathrm{va}}, C_{\mathrm{vf}}$ and $C_{\mathrm{vr}}$ are the mass specific heat at constant volume for air, fuel and residual gases, respectively.

The mass specific heat at constant pressure for the working fluid $\left(C_{\mathrm{pt}}\right)$ is defined as:

$$
\begin{aligned}
& C_{\mathrm{pt}}= \\
& \frac{\left(1-x_{\mathrm{r}}\right)\left[\left(\frac{m_{\mathrm{a}}}{m_{\mathrm{f}}}\right)_{\mathrm{s}} C_{\mathrm{pa}}+\phi C_{\mathrm{pf}}\right]+x_{\mathrm{r}} C_{\mathrm{pr}}\left[\phi+\left(\frac{m_{\mathrm{a}}}{m_{\mathrm{f}}}\right)_{\mathrm{s}}\right]}{\phi+\left(\frac{m_{\mathrm{a}}}{m_{\mathrm{f}}}\right)_{\mathrm{s}}},
\end{aligned}
$$

where $C_{\mathrm{pa}}, C_{\mathrm{pf}}$, and $C_{\mathrm{pr}}$ are the mass specific heat at constant pressure for air, fuel and residual gases, respectively.

The heat added per second in the isochoric $(2 \rightarrow 3)$ heat addition process may be written as

$$
\begin{aligned}
& \dot{Q}_{\mathrm{in}}= \\
& \dot{m}_{\mathrm{t}} C_{\mathrm{vt}}\left(T_{3}-T_{2}\right)=\dot{m}_{\mathrm{f}}\left\{\left(1-x_{\mathrm{r}}\right)\left[\left(\frac{m_{\mathrm{a}}}{m_{\mathrm{f}}}\right)_{\mathrm{s}} C_{\mathrm{va}}+\phi C_{\mathrm{vf}}\right]\right. \\
& \left.\quad+x_{\mathrm{r}} C_{\mathrm{vr}}\left[\phi+\left(\frac{m_{\mathrm{a}}}{m_{\mathrm{f}}}\right)_{\mathrm{s}}\right]\right\}\left(T_{3}-T_{2}\right) /\left[\phi\left(1-x_{\mathrm{r}}\right)\right],
\end{aligned}
$$

where $T$ is the absolute temperature.

The heat rejected in the isobaric heat rejection process $(5 \rightarrow 1)$ may be written as

$$
\begin{gathered}
\dot{Q}_{\mathrm{out}}=\dot{m}_{\mathrm{t}} C_{\mathrm{vt}}\left(T_{4}-T_{5}\right)+\dot{m}_{\mathrm{t}} C_{\mathrm{pt}}\left(T_{5}-T_{1}\right) \\
=\dot{m}_{\mathrm{f}}\left\{\left(1-x_{\mathrm{r}}\right)\left[\left(\frac{m_{\mathrm{a}}}{m_{\mathrm{f}}}\right)_{\mathrm{s}} C_{\mathrm{va}}+\phi C_{\mathrm{vf}}\right]+x_{\mathrm{r}} C_{\mathrm{vr}}\left[\phi+\left(\frac{m_{\mathrm{a}}}{m_{\mathrm{f}}}\right)_{\mathrm{s}}\right]\right\} \\
\left(-\gamma_{\mathrm{t}} T_{1}+T_{4}+\gamma_{\mathrm{t}} T_{5}-T_{5}\right) /\left[\phi\left(1-x_{\mathrm{r}}\right)\right],
\end{gathered}
$$

where $\gamma_{\mathrm{t}}$ is the specific heat ratio for the working fluid.

The total energy of the fuel per second input into the engine, of a gasoline type fuel, such as octane, can be expressed in terms of equivalence ratio factor from measured data as [5, 18-20]:

$$
\begin{aligned}
& Q_{\text {fuel }}=\eta_{\text {com }} \dot{m}_{\mathrm{f}} Q_{\mathrm{LHV}} \\
& =\left(-1.44738+4.18581 / \phi-1.86876 / \phi^{2}\right) \dot{m}_{\mathrm{f}} Q_{\mathrm{LHV}},
\end{aligned}
$$

where $\eta_{\text {com }}$ is the combustion efficiency and $Q_{\mathrm{LHV}}$ is the lower calorific value of the fuel. The range of effective $\phi$ values spans normal spark ignition combustion, i.e., from about 0.83 to about 1.33 [20].

The heat loss through the cylinder wall is given in the following linear expression [13-15, 18]:

$$
Q_{\mathrm{ht}}=\frac{\dot{m}_{\mathrm{f}} B\left[\phi+\left(\frac{m_{\mathrm{a}}}{m_{\mathrm{f}}}\right)_{\mathrm{s}}\right]\left(T_{2}+T_{3}-2 T_{0}\right)}{\phi\left(1-x_{\mathrm{r}}\right)},
$$

where $B$ is constant and $T_{0}$ is the absolute temperature.

Since the total energy of the delivered fuel $Q_{\text {fuel }}$ is assumed to be the sum of the heat added to the working fluid $Q_{\text {in }}$ and the heat leakage $Q_{\mathrm{ht}}$ :

$$
\begin{aligned}
Q_{\text {in }} & =Q_{\text {fuel }}-Q_{\mathrm{ht}} \\
= & \left(-1.44738+4.18581 / \phi-1.86876 / \phi^{2}\right) \dot{m}_{\mathrm{f}} Q_{\mathrm{LHV}} \\
& -\frac{\dot{m}_{\mathrm{f}} B\left[\phi+\left(\frac{m_{\mathrm{a}}}{m_{\mathrm{f}}}\right)_{\mathrm{s}}\right]\left(T_{2}+T_{3}-2 T_{0}\right)}{\phi\left(1-x_{\mathrm{r}}\right)} .
\end{aligned}
$$

The expansion-compression ratio, $\psi$, the effective compression ratio, $r_{\mathrm{c}}^{*}$, and the compression ratio, $r_{\mathrm{c}}$, are defined as:

$$
\begin{aligned}
& \psi=\frac{V_{5}}{V_{1}}=\frac{T_{5}}{T_{1}}, \\
& r_{\mathrm{c}}^{*}=\frac{V_{1}}{V_{2}},
\end{aligned}
$$

and

$$
r_{\mathrm{c}}=\frac{V_{5}}{V_{2}}=\frac{T_{5}}{T_{1}} r_{\mathrm{c}}^{*}=\psi r_{\mathrm{c}}^{*} .
$$

For the process $1 \rightarrow 2$, one finds that

$$
T_{2}=T_{1}\left(r_{\mathrm{c}}^{*}\right)^{\gamma_{\mathrm{t}}-1} \text {. }
$$

The entropy change $\Delta S_{3 \rightarrow 2}$ between states 2 and 3 , is equal to the entropy $\Delta S_{4 \rightarrow 1}$ change between states 4 and 1. Thus [16] 


$$
\begin{aligned}
& \Delta S_{3 \rightarrow 2}=\Delta S_{4 \rightarrow 1}=\Delta S_{4 \rightarrow 5}+\Delta S_{5 \rightarrow 1}, \\
& \mathrm{~d} S=C_{\mathrm{vt}} \frac{\mathrm{d} T}{T}+R \frac{\mathrm{d} V}{V} \text { or } \mathrm{d} S=C_{\mathrm{pt}} \frac{\mathrm{d} T}{T}-R \frac{\mathrm{d} P}{P} .
\end{aligned}
$$

Processes $2 \rightarrow 3$ and $4 \rightarrow 5$ occur at constant volume and $5 \rightarrow 1$ is a constant-pressure process. By substituting the specific heat from Eqs. (4) and (5) and integrating from the initial to the final state of the process, then

$$
\ln \left(\frac{T_{3}}{T_{2}}\right)-\ln \left(\frac{T_{4}}{T_{5}}\right)-\gamma_{\mathrm{t}} \ln \left(\frac{T_{5}}{T_{1}}\right)=0 .
$$

Taking into account the friction loss of the piston and assuming a dissipation term represented by a friction force that is a linear function of the piston velocity gives [5, 13]:

$$
f_{\mu}=-\mu S_{\mathrm{p}}=-\mu \frac{\mathrm{d} x}{\mathrm{~d} t},
$$

where $\mu$ is the coefficient of friction, which takes into account the global losses, $S_{\mathrm{p}}$ is the piston's velocity and $x$ is the piston's displacement. Therefore, the lost power due to friction is

$$
P_{\mu}=\frac{\mathrm{d} W_{\mu}}{\mathrm{d} t}=-\mu\left(\frac{\mathrm{d} x}{\mathrm{~d} t}\right)^{2}=-\mu\left(S_{\mathrm{p}}\right)^{2} .
$$

Running at $N$ revolutions per second, the mean velocity of the piston is $[13,19]$ :

$$
\bar{S}_{\mathrm{p}}=2 L N=2 L^{*}\left(\frac{r_{\mathrm{c}}^{*} \psi-1}{r_{\mathrm{c}}^{*}-1}\right) N,
$$

where $L$ is the total distance the piston travels per cycle and $L^{*}$ is the length of the isentropic process $1 \rightarrow 2$.

The power output of the Miller cycle engine is expressed by

$$
\begin{aligned}
& P_{\text {out }}=\frac{W}{\tau}-\left|P_{\mu}\right|=\dot{m}_{\mathrm{f}} \\
& \quad \times\left\{\left(1-x_{\mathrm{r}}\right)\left[\left(\frac{m_{\mathrm{a}}}{m_{\mathrm{f}}}\right)_{\mathrm{s}} C_{\mathrm{va}}+\phi C_{\mathrm{vf}}\right]+x_{\mathrm{r}} C_{\mathrm{vr}}\left[\phi+\left(\frac{m_{\mathrm{a}}}{m_{\mathrm{f}}}\right)_{\mathrm{s}}\right]\right\} \\
& \quad \times\left[\gamma_{\mathrm{t}} T_{1}-T_{2}+T_{3}-T_{4}+T_{5}-\gamma_{\mathrm{t}} T_{5}\right] / \phi\left(1-x_{\mathrm{r}}\right) \\
& \quad-4 \mu\left[L^{*} N\left(r_{\mathrm{c}}^{*} \psi-1\right) /\left(r_{\mathrm{c}}^{*}-1\right)\right]^{2} .
\end{aligned}
$$

The efficiency of the cycle is

$$
\begin{gathered}
\eta_{\mathrm{th}}=\frac{P_{\text {out }}}{Q_{\mathrm{in}}} \times 100=\left[-4 \mu \phi\left(1-x_{\mathrm{r}}\right)\left[L^{*} N\left(r_{\mathrm{c}}^{*} \psi-1\right)\right]^{2}\right. \\
/\left(\dot{m}_{\mathrm{f}}\left[r_{\mathrm{c}}^{*}-1\right]^{2}\left(T_{3}-T_{2}\right)\right. \\
\left.\times\left\{\left(1-x_{\mathrm{r}}\right)\left[\left(\frac{m_{\mathrm{a}}}{m_{\mathrm{f}}}\right)_{\mathrm{s}} C_{\mathrm{va}}+\phi C_{\mathrm{vf}}\right]+x_{\mathrm{r}} C_{\mathrm{vr}}\left[\phi+\left(\frac{m_{\mathrm{a}}}{m_{\mathrm{f}}}\right)_{\mathrm{s}}\right]\right\}\right) \\
\left.+\frac{\gamma_{\mathrm{t}} T_{1}-T_{2}+T_{3}-T_{4}+T_{5}-\gamma_{\mathrm{t}} T_{5}}{T_{3}-T_{2}}\right] \times 100
\end{gathered}
$$

When $r_{\mathrm{c}}^{*}$ and $T_{1}$ are given, $T_{2}$ can be obtained from Eq. (14). $\quad T_{3}$ can be deduced by substituting Eq. (6) into Eq. (10). $T_{5}$ can be found from Eq. (11), and $T_{4}$ can be obtained from Eq. (17). Substituting $T_{1}, T_{2}, T_{3}, T_{4}$, and $T_{5}$ into Eqs. (21) and (22), respectively, the power output and the thermal efficiency of the Miller cycle engine can be obtained. Therefore, the relations between the power output, the thermal efficiency and the compression ratio can be derived.

\section{Results and discussion}

The effects of equivalence ratio and mean piston speed on the performance of the dual cycle with $Q_{\mathrm{LHV}}=$ $44000 \mathrm{~kJ} \mathrm{~kg}^{-1}, \psi=1.25, L^{*}=10 \mathrm{~cm}, N=4500 \mathrm{rpm}$, $C_{\mathrm{vf}}=1.638 \mathrm{~kJ} \mathrm{~kg}^{-1} \mathrm{~K}^{-1}, C_{\mathrm{va}}=0.717 \mathrm{~kJ} \mathrm{~kg}^{-1} \mathrm{~K}^{-1}$, $C_{\mathrm{pf}}=1.7108 \mathrm{~kJ} \mathrm{~kg}^{-1} \mathrm{~K}^{-1}, C_{\mathrm{pa}}=1.004 \mathrm{~kJ} \mathrm{~kg}^{-1} \mathrm{~K}^{-1}$, $C_{\mathrm{vr}}=0.8268 \mathrm{~kJ} \mathrm{~kg}^{-1} \mathrm{~K}^{-1}, C_{\mathrm{pr}}=1.1335 \mathrm{~kJ} \mathrm{~kg}^{-1} \mathrm{~K}^{-1}$ $\left(m_{\mathrm{a}} / m_{\mathrm{f}}\right)_{\mathrm{s}}=14.6, \mu=12.9 \mathrm{~N} \mathrm{~s} \mathrm{~m}^{-1}, T_{0}=350 \mathrm{~K}$, $r_{\mathrm{c}}^{*}=1.4 \rightarrow 46, x_{\mathrm{r}}=10 \% \rightarrow 40 \%, T_{1}=400 \mathrm{~K}$, $\phi=0.85 \rightarrow 1.3, \dot{m}_{\mathrm{f}}=0.0025 \mathrm{~kg} \mathrm{~s}^{-1}, \phi=0.85 \rightarrow 1.3$ and $B=0.8 \mathrm{~kJ} \mathrm{~kg}^{-1} \mathrm{~K}^{-1}[5,11,16,21,22]$ are shown in Figs. 2-6. Using the above constants and range of parameters, the power output versus compression ratio characteristic and the power output versus thermal efficiency characteristic can be plotted.

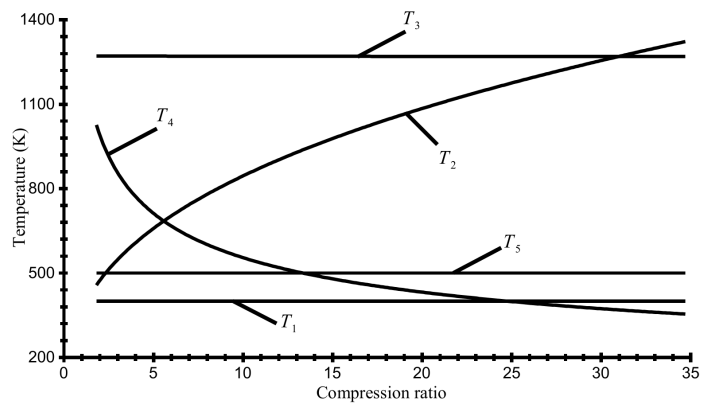

Fig. 2. The temperature versus compression ratio $\left(x_{\mathrm{r}}=40 \%, \phi=1\right)$.

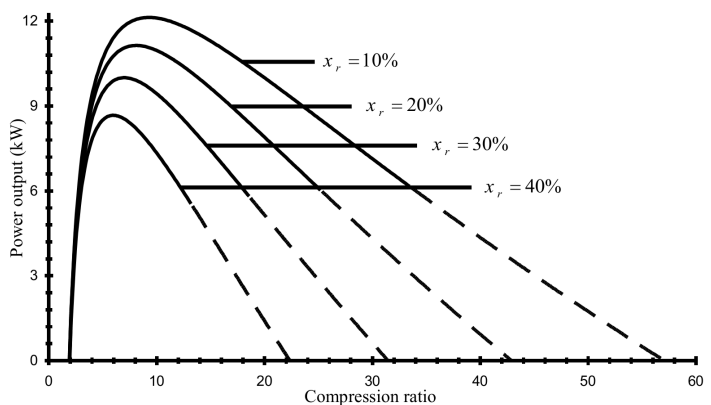

Fig. 3. Effect of residual gases on the variation of the power output with compression ratio $(\phi=1)$.

The variations in the temperatures $T_{2}, T_{3}, T_{4}$, and $T_{5}$ with the compression ratio are shown in Fig. 2. It is found that $T_{3}$ and $T_{4}$ decrease with increasing compression ratio, $T_{2}$ increases with increasing compression ratio and $T_{5}$ is constant with increasing compression ratio. In Fig. 2, there is one special state $\left(T_{5} \geq T_{4}\right)$. In this special state, the cycle cannot operate normally.

As it can be clearly seen from Eqs. (21) and (22), the power output and the thermal efficiency of the Miller cycle are dependent on the residual gases and the equivalence ratio. In order to illustrate the effects of these parameter, the relations between the power output and 


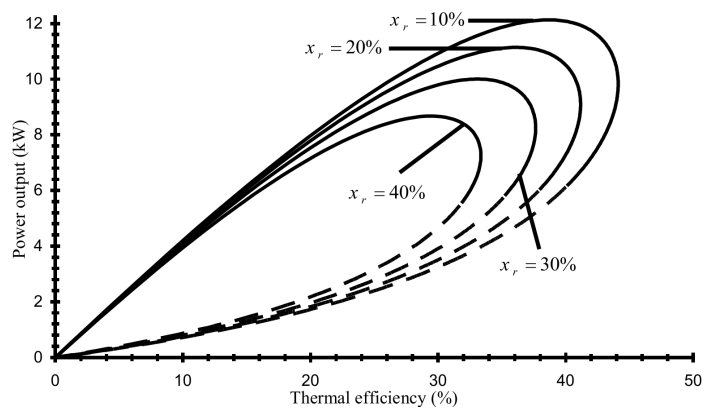

Fig. 4. Effect of residual gases on the variation of the power output with thermal efficiency $(\phi=1)$.

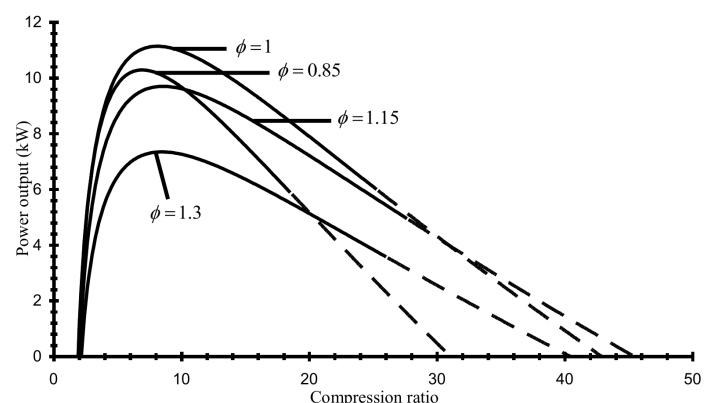

Fig. 5. Effect of equivalence ratio on the variation of the power output with compression ratio $\left(x_{\mathrm{r}}=20 \%\right)$.

the compression ratio, between the power output and the thermal efficiency of the cycle are presented in Figs. 3-6. It is found from these figures that the residual gases and the equivalence ratio play important roles on the performance cycle. They show that the maximum power output point and the maximum efficiency point are very adjacent. It is clearly seen from these figures that the effects of residual gases and equivalence ratio on the power output is related to compression ratio. They reflect the performance characteristics of a real irreversible Miller cycle engine. The power output versus compression ratio characteristic is approximately parabolic like curve. In other words, the power output increases with the in-

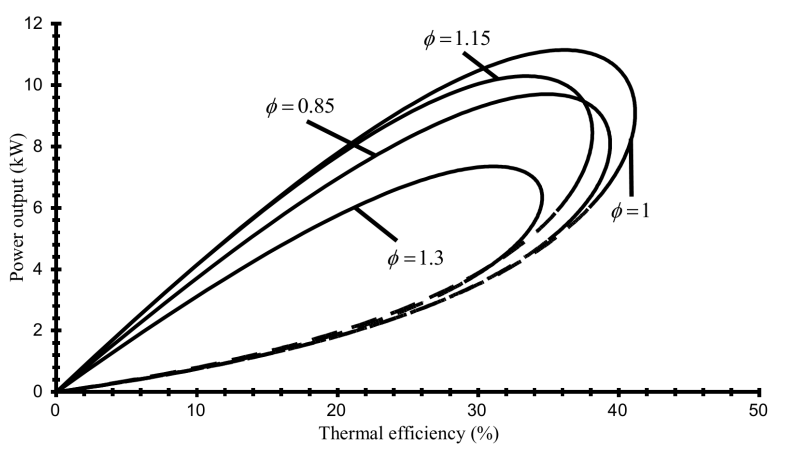

Fig. 6. Effect of equivalence ratio on the variation of the power output with thermal efficiency $\left(x_{\mathrm{r}}=20 \%\right)$. creasing compression ratio, attains its maximum value and then decreases with further increases in compression ratio. It should be noted that the heat added and the heat rejected by the working fluid decrease as the compression ratio increases. It can be seen that the curves of power output versus thermal efficiency are loop shaped as is common to almost all real heat engines [2, 23].

Figure 3 shows clearly that, throughout the compression ratio range, the power output decreases when the residual gases increased. This is due to the increase of the heat added by the working fluid being more than the increase of the heat rejected by the working fluid. In other words, the difference between heat added and heat rejected increases with increasing the cylinder wall temperature. This result is consistent with the experimental results in the internal combustion engine $[19,24,25]$. It can be seen from Figs. 3 and 4 that the maximum power output, the working range of the cycle, the compression ratio at the maximum power output, the efficiency at the maximum power output point and the power output at the maximum efficiency point decrease, when residual gases increase.

Referring to Fig. 5, it can be concluded that if compression ratio is less than certain value, the power output decreases with the increasing equivalence ratio. This can be attributed to the fact that the difference between heat added and heat rejected decrease with the increasing equivalence ratio. But if compression ratio exceeds certain value, the power output first increases and then starts to decrease with the increasing equivalence ratio. This can be attributed to the fact that the difference between heat added and heat rejected first increases and then starts to decrease with the increasing equivalence ratio. It can also be concluded that, for example, the power output at compression ratio of 9.6 first increases and then decreases as the equivalence ratio is increased. This result is consistent with the experimental results in the internal combustion engine [24, 25]. Figures 5 and 6 show that the optimal compression ratio corresponding to maximum power output point, the working range of the cycle, the thermal efficiency at the maximum power output point, the maximum power output, and the power output at the maximum efficiency point increase and then decrease as the equivalence ratio increases. Numerical calculation shows that for any same compression ratio, the smallest power output is for $\phi=1.3$ when $r_{\mathrm{c}}<20.2$ and is for $\phi=0.85$ when $r_{\mathrm{c}} \geq 20.2$ and also the largest power output is for $\phi=0.85$ when $r_{\mathrm{c}} \leq 2.9$, is for $\phi=1$ when $2.9<r_{\mathrm{c}} \leq 30.8$ and is for $\phi=1.1$ when $r_{\mathrm{c}}>30.8$.

According to above analysis, it can be found that the effects of the residual gases and the equivalence ratio on the cycle performance are obvious, and they should be considered in practice cycle analysis in order to make the cycle model be more close to practice. 


\section{Conclusion}

In this paper, an irreversible air standard Miller cycle model which is closer to practice is established. The relations between power output, thermal efficiency, compression ratio, residual gases and equivalence ratio are derived. The maximum power output and the corresponding efficiency and the maximum efficiency and the corresponding power output are also calculated. The detailed effect analyses are shown by numerical examples. The analysis helps us to understand the strong effects of residual gases and equivalence ratio on the performance of the Miller cycle. The general conclusions drawn from the results of this work are as follows:

- Throughout the compression ratio range, the power output increases with increasing residual gases.

- The maximum power output, the working range of the cycle, the compression ratio at the maximum power output, the efficiency at the maximum power output point, and the power output at the maximum efficiency point decrease, when cylinder wall temperature increases.

- If compression ratio is less than certain value, the power output decreases with increasing equivalence ratio, while if compression ratio exceeds certain value, the power output first increases and then starts to decrease with increasing equivalence ratio.

- The optimal compression ratio corresponding to maximum power output point, the working range of the cycle, the thermal efficiency at the maximum power output point, the maximum power output, and the power output at the maximum efficiency point increase and then decrease as the equivalence ratio increases.

This paper provides an additional criterion for use in the evaluation of the performance and the suitability of a Miller engine.

\section{References}

[1] L. Chen, F. Sun, Advances in Finite Time Thermodynamics: Analysis and Optimization, Nova Science Publishers, New York 2004.
[2] L. Chen, C. Wu, F. Sun, J. Non-Equilib. Thermodyn. 24, 327 (1999).

[3] R. Ebrahimi, Acta Phys. Pol. A 117, 887 (2010).

[4] P.Y. Wang, S.S. Hou, Energy Convers. Man. 46, 2637 (2005).

[5] R. Ebrahimi, Acta Phys. Pol. A 118, 534 (2010).

[6] Y. Ust, B. Sahin, A. Safa, Acta Phys. Pol. A 120, 412 (2011).

[7] R.H. Miller, ASME Trans. 69, 453 (1947).

[8] U. Kesgin, Int. Energy Res. 29, 189 (2005).

[9] K. Hatamura, M. Hayakawa, T. Goto, M. Hitomi, JSAE Rev. 18, 101 (1997).

[10] Y. Fukuzawa, H. Shimoda, Y. Kakuhama, H. Endo, K. Tanaka, Techn. Rev. 38, 146 (2001).

[11] A. Al-Sarkhi, B.A. Akash, J.O. Jaber, M.S. Mohsen, E. Abu-Nada, Int. Comm. Heat Mass Transfer $\mathbf{2 9}$, 1159 (2002).

[12] C. Wu, P.V. Puzinauskas, J.S. Tsai, Appl. Therm. Eng. 23, 511 (2003).

[13] Y. Ge, L. Chen, F. Sun, C. Wu, Int. Comm. Heat Mass Transfer 32, 1045 (2005).

[14] Y. Ge, L. Chen, F. Sun, C. Wu, J. Appl. Energy 81, 397 (2005).

[15] Y. Ge, L. Chen, F. Sun, C. Wu, Int. J. Ambient Energy 26, 203 (2005).

[16] A. Al-Sarkhi, J.O. Jaber, S.D. Probert, J. Appl. Energy 83, 343 (2006).

[17] L. Chen, W. Zhang, F. Sun, J. Appl. Energy 84, 512 (2007).

[18] R. Ebrahimi, Appl. Math. Model. 36, 4073 (2012).

[19] J.B. Heywood, Internal Combustion Engine Fundamentals, McGraw-Hill, New York 1988.

[20] G.H. Abd Alla, Energy Convers. Man. 43, 1043 (2002).

[21] Y. Ust, B. Sahin, O.S. Sogut, J. Appl. Energy 82, 23 (2005).

[22] S.S. Hou, J.C. Lin, Acta Phys. Pol. A 120, 979 (2011).

[23] J.M. Gordon, M. Huleihil, J. Appl. Phys. 72, 829 (1992).

[24] M. Mercier, Ph.D. Thesis, Université de Valenciennes et du Hainaut-Cambrésis, France 2006 (in French).

[25] A. Hocine, Ph.D. thesis, Université de Valenciennes et du Hainaut-Cambrésis, France 2003. 